Nádia Zanon Narchi ${ }^{1}$ Elizabete Franco Cruz ${ }^{1}$ Roselane Gonçalves ${ }^{1}$

\section{O papel das obstetrizes e enfermeiras obstetras na promoção da maternidade segura no Brasil}

\author{
The role of midwives and nurse-midwives \\ in promoting safe motherhood in Brazil
}

\begin{abstract}
Brazil needs to improve maternal and perinatal health care, given the high rates of morbidity, mortality and cesarean sections, the medicalization process of the female body and the violation of the sexual and reproductive rights of women during pregnancy and childbirth. Based on the contribution of international researchers and agencies to address these issues, the scope of this text is to provide theoretical input to support the proposal of the promotion of safe motherhood that requires the active participation of midwives and nurse-midwives. These are professionals who, from the perspective of health promotion, can collaborate in the establishment of an effective network of care, whose basic premise is the humanization of care based on scientific evidence in order to improve the experience of women and the family in the process of pregnancy, childbirth and postpartum.
\end{abstract}

Key words Maternal mortality, Perinatal mortality, Obstetrical nursing, Midwife, Health poli$c y$, Professional training
Resumo O Brasil necessita melhorar a assistência à saúde materna e perinatal, haja vista os indices elevados de morbimortalidade e de cesáreas, o processo de medicalização do corpo feminino e a violação dos direitos sexuais e reprodutivos das mulheres durante o ciclo gravídico-puerperal. A partir da contribuição de pesquisadores e organismos internacionais dedicados ao estudo destas questões, este texto tem como finalidade oferecer subsídios teóricos que sustentem a proposta de que a promoção da maternidade segura requer a efetiva participação de obstetrizes e enfermeiras obstetras, profissionais que, a partir da perspectiva da promoção da saúde, podem colaborar na constituição de uma rede efetiva de cuidados, cuja premissa básica seja a atenção humanizada e baseada em evidências científicas a fim de melhorar a experiência da mulher e da família no processo de gestação, parto e pós-parto.

Palavras-chave Mortalidade materna, Mortalidade perinatal, Enfermagem obstétrica, Obstetriz, Política de saúde, Capacitação profissional
${ }^{1}$ Curso de Obstetrícia, Escola de Artes, Ciências e Humanidades,

Universidade de São Paulo. Av. Arlindo Bettio 1.000/ 202-C, Ermelino

Matarazzo. 03828-000 São Paulo SP.nzn@usp.br 


\section{Saúde materna e perinatal: contexto e desafios}

Para alcançar o quinto objetivo de desenvolvimento do milênio (ODM) o Brasil deve promover ações, entre 1990 e 2015, que resultem na redução em três quartas partes da mortalidade materna, e que promovam o alcance, até 2015, do acesso universal à saúde reprodutiva.

Dentre as linhas de cuidado prioritárias definidas em 2011 pelo Ministério da Saúde (MS) a fim de melhorar o quadro da saúde materna, destaca-se a proposta de qualificar profissionais para promover atenção obstétrica e neonatal humanizada e baseada em evidências científicas. Por sua vez, a qualificação da atenção compreenderá a criação de novas estruturas de assistência e acompanhamento das mulheres na atenção primária, nos serviços de alto risco e de urgências obstétricas, e na rede hospitalar convencional, que deverá contar com Casas da Gestante e do Bebê e com Centros de Parto Normal, extra ou intra-hospitalares.

Este texto propõe-se a fornecer subsídios teóricos para a inserção de parteiras, diplomadas ou profissionais, graduadas, habilitadas na promoção da maternidade segura. A partir de dados de especialistas mundiais e nacionais de mortalidade materna pretende-se analisar quais elementos relacionados ao contexto e à qualificação profissional necessitam ser reparados a fim de possibilitar melhorias no cenário brasileiro da atenção à saúde materna e perinatal.

Relatório do ano de 2010 da Organização Mundial da Saúde (OMS) ${ }^{1}$ afirma que complicações da gravidez, parto e pós-parto matam mais de meio milhão de mulheres ao ano em todo o mundo, havendo cerca de outros $10 \mathrm{mi}-$ lhões que ficam com sequelas das complicações sofridas no período. Apesar dessa situação e de imensas diferenças entre os países, a OMS destaca que existem sinais de progresso, mesmo em áreas mais críticas, com mais mulheres em idade reprodutiva ganhando acesso a cuidados prestados por profissionais de saúde.

Todavia, a Anistia Internacional, em documento denominado "Parto Mortal: a crise na saúde materna e infantil nos Estados Unidos"2, indica que a mortalidade materna duplicou nos Estados Unidos nos últimos 20 anos, analisando que mesmo os países ricos em recursos não têm adotado práticas que tratam as mulheres com dignidade, respeito e atendimento apropriado durante a gestação, o parto e o pós-parto.

Publicação do Conselho de Direitos Humanos das Nações Unidas ${ }^{3}$ relata que a mortalidade materna evitável é uma questão premente que viola os direitos humanos da mulher à saúde, à vida, à educação, à dignidade e à informação. $\mathrm{Na}$ mesma linha de argumentação, documento da Organização dos Estados Americanos (OEA) ${ }^{4}$ destaca que a mortalidade materna é um indicador de disparidade e desigualdade entre homens e mulheres, sendo sua extensão um claro sinal de discriminação contra a mulher, que encontra maiores dificuldades no acesso a serviços, saúde, nutrição e oportunidades econômicas, não havendo, no caso dos homens, uma única causa de morte ou de incapacidade cuja magnitude se aproxime de todos os casos de morbimortalidade materna.

O citado relatório da $\mathrm{OMS}^{1}$, que descreve tendências da mortalidade materna no período de 1990 a 2008 , mostra que a média mundial na razão de mortalidade materna (RMM) caiu de 400 para 260 óbitos por 100.000 nascidos vivos (NV) no período. Os países que menos conseguiram avançar na erradicação do problema foram os da Oceania e da África, enquanto os da Ásia e da América Latina e Caribe apresentaram reduções importantes.

No que tange ao Brasil, a meta do país é reduzir a RMM até 2015 a 35 óbitos de mulheres a cada 100.000 NV. Todavia, esse índice, que caiu de 140 em 1990 a 75 em 2007, tem-se mantido estável e sempre superior a 70 desde o ano $2000^{5}$, o que leva a crer que o quinto ODM dificilmente será alcançado.

Relatório do MS do ano de $2009^{5}$, que analisa a situação da saúde no Brasil, ressalta que mais da metade das mortes maternas e neonatais ocorrem durante a internação da mulher para o parto e nascimento; cerca de $70 \%$ das mortes das mulheres ocorrem por causas obstétricas diretas; por volta de $15 \%$ das mortes são decorrentes de aborto inseguro; $51 \%$ das mortes neonatais ocorrem na primeira semana de vida e se relacionam majoritariamente com problemas decorrentes do parto; para cada morte materna, outras trinta mulheres sofrem sequelas ou problemas crônicos de saúde decorrentes de complicações ocorridas durante a gravidez, parto ou pós-parto.

Em dezembro de 2010, durante encontro de perinatologia, cujo tema central foi a "Epidemia de Prematuros", debateu-se que os nascimentos pré-termo estão aumentando rapidamente em todo o país. Este é um dos motivos que leva os profissionais da área a questionar a definição de parto a termo para crianças nascidas entre 37 e 38 semanas de gestação, cuja morbimortalidade é elevada em comparação àquelas nascidas a partir das 39 semanas. Os erros na estimativa, 
pela data da última menstruação ou pelo ultrassom, mesmo aquele realizado precocemente, mostram que frequentemente ocorre superestimação em 10 a 14 dias na idade gestacional. Este fato, somado ao aumento progressivo dos índices de cesárea eletiva, tem ocasionado repercussão direta sobre a mortalidade neonatal precoce e sobre os índices crescentes de prematuridade e de baixo peso ao nascer.

Em relação à cirurgia cesariana, o Sistema Único de Saúde (SUS) brasileiro registra aumento no seu percentual, de $37,8 \%$ em 2000 para $46,5 \%$ em 2009. Esse elevado índice, que chega a $90 \%$ no setor de saúde suplementar, tem proporcionado ao nosso país o título de campeão mundial na realização de cesáreas eletivas, bem como contribuído para aumento no percentual de nascimentos de bebês com baixo peso ${ }^{5,7}$.

É fato que a mortalidade materna tem estreita relação com a discriminação e com os determinantes biológicos e socioculturais das mulheres brasileiras, em especial com as desigualdades de gênero. Cabe ressaltar também que a qualidade da assistência interfere diretamente na questão. Nesta perspectiva, pode-se afirmar que a realidade brasileira se constitui em um mosaico de evolução, estagnação e regressão.

Evolução, porque não faltam no Brasil programas, políticas, leis, isto é, incentivos oficiais e tentativas de regulação dos serviços públicos e privados de saúde. São exemplos desse investimento: o Projeto Maternidade Segura de 1996; o Programa de Humanização do Pré-natal e Nascimento (PHPN) de 2000; o Pacto Nacional pela Redução da Mortalidade Materna e Neonatal de 2002; a Política Nacional de Atenção Integral à Saúde da Mulher de 2003, que inclui a atenção ao abortamento em condições inseguras e a redução da mortalidade materna; o Plano de Qualificação de Maternidades e Redes Perinatais de 2010; o Projeto Rede Cegonha; Resoluções da Agência Nacional de Saúde Suplementar e da Agência Nacional de Vigilância Sanitária; a Lei do Acompanhante, de 2005, outro importante dispositivo de destaque na promoção da maternidade segura.

Ressaltam-se nesse contexto evolutivo a ampliação da cobertura pré-natal e do uso e oferta de contraceptivos; a alta cobertura institucional para o parto; o investimento do MS na capacitação profissional de médicos e enfermeiros; o envolvimento da sociedade civil organizada na reinvindicação do pleno exercício dos direitos sexuais e reprodutivos das mulheres, ressaltando o direito de escolha do tipo de parto e da autonomia da mulher; a mudança do modelo de cuidado prestado à mulher, ao recém-nascido e à sua família, o que necessariamente inclui alterações estruturais, funcionais e de recursos humanos. A criação de Centros de Parto Normal extra ou intra-hospitalares, por exemplo, advém dessa mudança conceitual e fundamenta-se na assistência humanizada, com base em evidências científicas e centrada na mulher.

Somente essas estratégias porém, conforme destacado, tem se mostrado insuficientes para melhorar a qualidade assistencial, pois a precariedade dos serviços públicos de saúde é marcan$\mathrm{te}^{5,8-10}$, havendo muito por fazer ou melhorar. A estagnação e, até mesmo, a regressão do processo, no qual os resultados satisfatórios não são ampliados a toda a sociedade, estabelece relação direta tanto com o contexto insatisfatório quanto com a inadequada qualificação dos profissionais de saúde.

O sucateamento do serviço público, que não conta com estrutura física, material e organizacional adequadas para promover qualidade assistencial às mulheres, recém-nascidos e famílias, e o caráter mercantilista e excludente do serviço privado, cujo foco é a assistência mais complexa e o uso de tecnologias desnecessárias e dispendiosas, tem contribuído para levar à escolha da cesárea como parto ideal. Esse cenário é recrudescido pela progressiva terceirização dos serviços públicos, o que fragiliza o SUS.

Fatores relacionados à violência institucional e à violação dos direitos da mulher e da criança, ao abuso do poder nas relações e à discriminação de gênero provocam iatrogenias e negligência no cuidado. Espaços que deveriam ter como foco central o cuidado das mulheres configuramse como cenários em que estas têm seus direitos à integridade física, psíquica e moral violados. Ilustra essa situação pesquisa realizada pela Fundação Perseu Abramo ${ }^{11}$ mostrando que 25\% das mulheres que tiveram filhos de parto natural na rede pública ou privada sofreram algum tipo de violência ao serem atendidas durante o trabalho de parto e parto.

Nessa perspectiva, observa-se que muitas mulheres não conhecem ou fazem valer seus direitos, submetendo-se ao sistema e ao profissional de saúde, especialmente em momentos cercados de temores e fragilidades, como são a gravidez, o parto e o pós-parto. O fraco controle social, as concepções biomédicas, a cultura da gestação como doença e do parto como evento perigoso, a falta de autonomia e a descrença na fisiologia e no poder do corpo feminino, provocam nas mulheres restrição de sua cidadania, o que dificulta o necessário processo de evolução e mudança social. 
Em relação ao pré-natal, verifica-se que o foco na ampliação do número de consultas por si só não tem garantido qualidade assistencial. Revisão da Cochrane ${ }^{12}$ não encontrou em gestantes de baixo risco diferença nos resultados maternos e perinatais com quatro ou seis consultas. Por esse motivo, recomenda-se que haja aprimoramento na qualidade das consultas e no acompanhamento individual e familiar das gestantes, para que ocorra de fato promoção de melhorias nos estilos de vida e saúde, detecção contínua do risco materno e perinatal, preparo para o parto, a maternidade, o aleitamento, o pós-parto, entre outros aspectos fundamentais da abordagem gestacional ${ }^{13-18}$.

Quanto ao pós-parto, trabalhos ${ }^{15-18}$ mostram que é frequente o inadequado acompanhamento e suporte da puérpera e da família no período, o que provoca o "quase abandono" da mulher à sua própria sorte, produzindo reflexos negativos em sua saúde e na do seu bebê.

A assistência hospitalar ao parto, especialmente nos serviços públicos, continua ocorrendo em ambientes desfavoráveis, sem privacidade, suporte e apoio de familiares, com uso de procedimentos rotineiros e sem base em evidências científicas ${ }^{18-20}$. Durante a evolução do parto não se observa a sua fisiologia, buscando-se, constantemente, correções do processo por meio da medicalização e do uso de intervenções que provocam consequências negativas sobre a saúde de mães e bebês.

Este é o cenário da assistência não qualifica$\mathrm{da}$, ou seja, nem sempre promovida por profissional capacitado e atualizado. Ela é prevalentemente tecnológica, muitas vezes iatrogênica e desrespeitadora dos direitos das mulheres e dos recém-nascidos, uma vez que pode provocar tanto mortalidade quanto morbidade física e psicossocial.

Cecatti ${ }^{21}$ destaca que, independentemente das condições biológicas e psicossociais das mulheres, apenas a melhora da atenção profissional e institucional é capaz de reduzir a ocorrência de morbidade grave e de mortes maternas. Vale, no contexto deste trabalho, citar pesquisas que descrevem as dificuldades encontradas pelas enfermeiras, nem todas especialistas em obstetrícia, para promover atenção obstétrica qualificada.

Os trabalhos de Bussadori ${ }^{22}$, Cagnin ${ }^{23}$, Cunha et al. ${ }^{16}$, Dotto et al. ${ }^{19,24}$ e Dotto e Mamede ${ }^{25}$, Esser $^{26}$, Fornazari ${ }^{27}$, Leite ${ }^{28}$, Narchi ${ }^{17,18,20}$, Noguei$\mathrm{ra}^{29}$, Sabino $^{30}$, e Vorpagel ${ }^{31}$ evidenciam, entre outros aspectos, que as enfermeiras apresentam déficit no saber e no saber-fazer em obstetrícia, o que indica falhas na sua formação, tanto na graduação quanto na especialização. Por esse motivo e por pressão institucional, elas frequentemente utilizam práticas desaconselhadas pelas evidências científicas, muitas delas prejudiciais, o que mostra a prevalência do modelo biomédico e intervencionista.

Praticamente todos os estudos supracitados concluem que as barreiras pessoais e institucionais com que se defrontam as enfermeiras impedem a assistência qualificada, havendo necessidade de investimento na melhor formação e na qualificação profissional permanente, bem como na reorganização dos serviços e na revisão e incorporação de protocolos assistenciais para a melhoria da qualidade.

Destaca-se que grande parte dos problemas levantados por essas pesquisas é enumerada em relatório do Fundo de Populações da Organização das Nações Unidas (UNFPA) ${ }^{32}$, que salienta a necessidade de os países investirem na formação de obstetrizes $(\mathrm{O})$ e/ou enfermeiras obstetras (EO) suficientemente habilitadas para promover atenção qualificada e maternidade segura. As barreiras citadas vão desde o desconhecimento da população acerca do papel dessas profissionais na atenção até ao número insuficiente delas e sua falta de capacitação e atualização teórica e clínica.

Outro documento, também da UNFPA, intitulado "Intensificação da capacidade das parteiras para reduzir a morbimortalidade materna" 33 , acrescenta mais uma importante barreira para a atenção qualificada: a discriminação ou a iniquidade de gênero com que se defrontam as trabalhadoras nos serviços de saúde. Como mulheres ou força de trabalho majoritariamente feminina, $\mathrm{O}$ e/ou EO enfrentam dificuldades que não aconteceriam caso fossem homens. Como são mulheres, trabalhando em parceria com as mulheres, valorizadas pelas mulheres que assistem, são ignoradas ou discriminadas pelo sistema.

\section{Caminhos para mudança: efetiva inserção de obstetrizes e enfermeiras obstetras na atenção}

Mudar o quadro da atenção à saúde materna e perinatal no Brasil exige diversas intervenções: qualidade da assistência voltada para as necessidades das mulheres, o que requer mudança na cultura dos serviços de saúde; ambiente acolhedor e favorável, o que exige reformas na estrutura dos serviços de saúde; garantia de participação à mulher no processo, exercendo sua auto- 
nomia de forma compartilhada, o que advirá de maior controle social, bem como enfoque na discriminação e na perspectiva de gênero; respeito à fisiologia do processo, o que requer transformação na atuação do profissional de saúde; e, finalmente, capacitação, reconhecimento e autonomia dos profissionais.

Nesse sentido, considera-se fundamental que haja inserção de maior número de O e EO aptas a exercer as competências essenciais em obstetrícia, isto é, capacitadas para o manejo adequado das situações obstétricas, de risco ou não. Evidências mostram que modelos de assistência envolvendo essas profissionais associam-se a menores taxas de intervenções e em maior satisfação das mulheres ${ }^{34-36}$.

Por este motivo, a OMS recomenda que haja incentivo na formação e qualificação de maior número de $\mathrm{O}$ e/ou EO, bem como a sua participação na assistência comunitária ${ }^{37}$. Além disso, faz-se importante destacar que práticas exitosas de outros países na redução da mortalidade materna e perinatal guardam estreita relação com a existência de grande número dessas profissionais e, mais que isso, um quadro social em que se distingam como profissões respeitadas e atraentes, capacitadas, regulamentadas e com ingresso garantido no sistema de saúde $e^{4,32,33,38,39}$.

O já citado documento da UNFPA ${ }^{33}$ declara que sem esforços governamentais para aumentar o número de $\mathrm{O}$ e/ou EO, as mulheres e seus bebês continuarão a morrer desnecessariamente, as mulheres continuarão a ter seus direitos sexuais e reprodutivos desrespeitados, a gestação e o parto continuarão a ser excessivamente medicalizados.

Corrobora com essa afirmação pesquisa realizada pela Universidade de Oxford, citada em relatório da $\mathrm{OEA}^{4}$, mostrando que nenhum país conseguiu reduzir a mortalidade materna sem investir na capacidade das $\mathrm{O}$ e/ou das $\mathrm{EO}$ em trabalhar na atenção primária ou comunitária, o que inclui a assistência à mulher e à família durante todo o ciclo gravídico-puerperal, bem como o parto em locais como o domicílio ou os centros de parto intra ou peri-hospitalares.

Além de contribuir para a melhoria assistencial, é importante ressaltar que $\mathrm{O}$ e/ou EO têm sido as principais responsáveis por produzir trabalhos de inegável contribuição para a mudança da prática obstétrica, haja vista as inúmeras publicações dessas profissionais em revisões sistemáticas da Cochrane.

Contudo, e conforme citado, diversos traba$\operatorname{lhos}^{18-20,22-31,40-45}$ têm mostrado problemas rela- cionados à formação e atuação das EO no Brasil, desde a falta de autonomia e visibilidade até a dificuldade para assumir um perfil estritamente assistencial. Por possuírem identidade híbrida, de enfermeira e de obstetriz, as enfermeiras habilitadas ou especialistas em obstetrícia muitas vezes não conseguem atuar na área específica, sendo deslocadas para outras funções e outros setores, mesmo a contragosto. Além disso, algumas EO apresentam baixa expectativa de atuação na assistência direta, revelando que sua simples especialização em obstetrícia nem sempre produz o necessário e esperado impacto na qualidade e na transformação do modelo de atenção ao processo de parto e nascimento.

Independentemente dos problemas apontados, faz-se importante salientar que sem as EO o movimento de humanização e de transformação do modelo assistencial ao parto e nascimento não teria avançado em nosso país; seu trabalho tem sido fundamental para o processo de mudança e só não é mais efetivo por falta de investimento e valorização do sistema de saúde e pelo número reduzido de profissionais técnica e politicamente atuantes.

A fim de produzir impacto na assistência, a $\mathrm{OMS}^{37}$ recomenda que os países devam ter, no mínimo, uma parteira qualificada para cada 125 nascimentos ao ano. Levando em conta essa estimativa, o Brasil deveria possuir ao menos 23.000 O e/ou EO, número bem aquém do existente. A Associação Brasileira de Obstetrizes e Enfermeiros Obstetras (ABENFO) estima que deve haver, no máximo, $10.000 \mathrm{EO}$ no país, não havendo nenhuma pesquisa que confirme esse dado e nem que aponte qual é o percentual de profissionais que atua na área da assistência obstétrica.

A relevância da formação de Obstetrizes se inscreve, portanto, no contexto da atual realidade brasileira. Aumentar o quantitativo de profissionais capacitados, incrementar sua qualificação, agregar valor à área, tornar mais específico o âmbito de sua atuação, mantendo a perspectiva de promoção da saúde integral, e pressionar pela transformação do modelo de atenção, são as principais justificativas que sustentam essa formação no Brasil, proposta defendida por diversas entidades e por pesquisadores envolvidos com a temática da saúde da mulher ${ }^{46-52}$.

Importantes documentos de organizações mundiais $^{4,32,33,38,39,53}$ mostram que a efetiva inserção de O e EO promove a melhoria da atenção obstétrica. Pesquisas e revisões sistemáticas dão claros sinais da relevância dessas profissionais em contextos assistenciais de outros países. 
No Brasil, existem várias pesquisas sobre a assistência integral à saúde da mulher. Entretanto, ainda são poucas as que evidenciam qual é o impacto provocado pela presença das $\mathrm{O}$ e EO na assistência obstétrica. Assim, para pensar sua inclusão na atenção às mulheres durante o ciclo gravídico-puerperal é preciso considerar o cenário no qual se inserem e a trajetória de ambas as profissões.

Foge ao escopo deste texto o aprofundamento de tais questões, mas é preciso entender que a formação de $\mathrm{O}$ e $\mathrm{EO}$ apresentou um importante hiato nas últimas décadas do século passado. Apenas no final dos anos 90 é que a Enfermagem Obstétrica sofreu um novo impulso ${ }^{46-52}$ e a formação de O só foi retomada no país em 2005. Pelo exposto, discutem-se profissões que muito recentemente têm buscado fortalecimento e reconhecimento num contexto dominado predominantemente por médicos e, mais do que isso, pelo modelo biomédico.

Distinguem-se nesse complexo emaranhado, problemas quanto à formação dos diferentes profissionais de saúde, o modelo de assistência e as práticas empregadas, a disputa por espaço nas instituições e nas políticas de saúde. Estes são provavelmente os motivos pelos quais no campo dos conhecimentos produzidos na prática cotidiana, encontram-se depoimentos de mulheres, profissionais e instituições que alimentam a certeza de que o Brasil precisa transformar a realidade da atenção obstétrica.

Ao se unirem as pesquisas sobre a saúde da mulher, a enfermagem e a obstetrícia, a avaliação de instituições nacionais e internacionais, os saberes da prática e a compreensão das duas profissões, observa-se que ambas se encontram diante de uma possibilidade que se sustenta em sua própria trajetória histórica e que precisam de espaço, tempo e investimento para se consolidar e desenvolver no país.

Nessa perspectiva, relatório da $\mathrm{UNFPA}^{53}$, denominado "O Estado das Parteiras no Mundo 2011”, exorta governos, órgãos de regulamentação profissional, escolas e instituições de capacitação, associações profissionais, organizações internacionais, alianças mundiais e entidades da sociedade civil organizada a se envolver e desenvolver ações a fim de fortalecer as profissões de "partería”, entre nós de O e de EO.

As contribuições desse documento são extremamente relevantes, pois apontam ações necessárias para que os países efetivamente incorporem $\mathrm{O}$ e/ou EO na assistência à saúde das mulheres. Este caminho inclui estratégias de ampliação do núme- ro de profissionais, fortalecimento das competências e valorização da formação profissional, regulamentação das profissões, fomento à produção de conhecimentos e incentivo à visibilidade.

O mencionado relatório da UNFPA destaca, ainda, um conjunto de disposições governamentais que devem ser tomadas com o intuito de: reconhecer essas duas profissões como distintas das demais da área da saúde e, ambas, essenciais para a provisão de serviços de saúde materna e perinatal, promovendo-as como carreiras no nível das políticas nacionais; assegurar que essas profissões sejam incorporadas aos programas de atenção à saúde materna e perinatal e que estejam harmonizadas com os planos de recursos humanos; assegurar uma adequada disponibilidade e distribuição de estabelecimentos de saúde que ofereçam atenção obstétrica e neonatal de urgência, incluindo maternidades e unidades de atenção administradas diretamente por $\mathrm{O}$ e/ou EO; e, por fim, investir em gestão de recursos humanos para desenvolver e manter as competências dessas profissionais, gerenciando e controlando o contingente necessário aos serviços de saúde.

Além disso, o citado documento aponta a necessária participação das organizações internacionais, alianças mundiais e entidades da sociedade civil organizada, no apoio a programas em nível local, regional e internacional a fim de: ampliar os serviços de atenção promovidos pelas O e EO, melhorando a medição de seus resultados; promover serviços de atenção obstétrica administrados por $\mathrm{O}$ e EO, fortalecidos com a melhoria das competências e a qualidade da atenção, incluindo os serviços nas estratégias e programas de saúde; fornecer apoio financeiro para o fomento das associações profissionais e a facilitação de intercâmbio de conhecimentos e de informações sobre as boas práticas e as inovações; e, finalmente, estabelecimento de uma agenda para a realização de estudos sobre essas profissões e o impacto produzido pela atenção que promovem.

\section{Considerações finais}

No Brasil, um dos desafios do momento é buscar estratégias efetivas de inserção e valorização de $\mathrm{O}$ e EO devidamente capacitadas para promover atenção humanizada e baseada em evidências científicas, cuja premissa básica seja a de estar com a mulher e a família, melhorando sua vivência no processo de gestação, parto e pósparto. 
Buscar parcerias, fortalecer órgãos de classe, unir esforços, atuar politicamente, refletir sobre a prática, atualizar-se continuamente, ser flexível e aceitar inovações, colaborar com outros agentes de saúde e com a comunidade, contribuir para a mudança do modelo de atenção, são elementos imprescindíveis para a devida valorização e inserção dessas profissionais na promoção da gravidez segura, no incentivo ao parto normal e seguro, na prática orientada por revisões sistemáticas de ensaios clínicos randomizados, no estar com a mulher de forma a garantir-lhe os direitos sexuais e reprodutivos e o devido suporte e apoio.

Considera-se fundamental conduzir a assistência obstétrica menos complexa de volta à co- munidade, provendo instalações adequadas e profissionais com as competências necessárias, o que significa dizer que urge criar maior número de Centros de Parto Normal extra ou intrahospitalares coordenados por O e EO, profissionais que, a partir da perspectiva da promoção da saúde, podem colaborar na constituição de uma rede mais efetiva de cuidados.

Sem dúvida, esse é um debate complexo e multifacetado, que envolve um cenário de disputas e desafios. Contudo, abster-se do enfrentamento dessa questão ou resistir às transformações, significa furtar-se do compromisso histórico de construir melhores alternativas para a saúde da mulher brasileira.

\section{Colaboradores}

NZ Narchi, EF Cruz e R Gonçalves participaram igualmente de todas as etapas de elaboração do artigo. 


\section{Referências}

1. World Health Organization (WHO). Trends in Ma ternal Mortality: 1990 to 2008. Geneva: WHO; 2010. [cited 2011 Jul 5]; [about 55 p.]. Available from: http://whqlibdoc.who.int/publications/2010/978924 1500265_eng.pdf

2. Amnesty International. USA: Deadly delivery: The maternal health care crisis in the USA. USA: Amnesty International; 2010. [cited $2011 \mathrm{Jul} 5$ ]; [about 154 p.]. Available from: http://www.amnesty.org/ en/library/asset/AMR51/007/2010/en/968e78f684be-4c3b-975f-170f21fe707c/amr510072010es.html

3. Organización de las Naciones Unidas (ONU). Consejo de Derechos Humanos de las Naciones. Mortalidad y morbilidad maternas prevenibles y derechos humanos. Washington: ONU; 2009. [asesado 2011 jul 5]; [cerca de 4 p.]. Disponible en: http://ap ohchr.org/documents/S/HRC/resolutions/A_HRC_ RES_11_8.pdf

4. Organización de los Estados Americanos (OEA). Acceso a servicios de salud materna desde una perspec tiva de derechos humanos. Washington: OEA; 2006. [asesado 2011 jul. 5]; [cerca de 36 p.]. Disponible en: http://cidh.org/women/SaludMaterna10Sp/Salud Materna2010.pdf

5. Brasil. Ministério da Saúde (MS). Saúde Brasil 2009 Uma análise da situação de saúde e da agenda nacional e internacional de prioridades em saúde. Brasília: MS; 2010. [acessado 2011 jul. 5]; [cerca de 49 p.]. Disponível em: http://portal.saude.gov.br/portal/arquivos/ pdf/SAUDE_BRASIL_2009_COLETIVA.pdf

6. Sociedade de Pediatria do Rio Grande do Sul (SPRS). Resumo Encontro de Perinatologia; 2010. [acessado 2011 jul. 5]; [cerca de 1 p.]. Disponível em: http://www.sprs.com.br/index.php?option= com_content\&task=view $\$$ ionid=\&id=8609\&Itemid=

7. Agência Nacional de Saúde Suplementar (ANS). O modelo de atenção obstétrica no setor de saúde suplementar no Brasil: cenários e perspectivas. Rio de Janeiro: ANS; 2008.

8. Rattner D. Humanização na atenção a nascimentos e partos: breve referencial teórico. Interface Comun Saúde Educ 2009; 13(Supl. 1):595-602.

9. Rattner D. Humanização na atenção a nascimentos e partos: ponderações sobre políticas públicas. In terface Comun Saúde Educ 2009; 13(Supl. 1):759-768

10. Diniz SG. Gênero, saúde materna e o paradoxo perinatal. Rev Bras Crescimento e Desenvolvimento Hum. 2009; 19(2):313-326.

11. Fundação Perseu Abramo. Gravidez, filhos e violência institucional no parto. Mulheres Brasileiras e Gênero nos Espaços Público e Privado. São Paulo: Fundação Perseu Abramo; 2010. [acessado 2011 jul 5]; [cerca de 301 p.]. Disponível em: http://www. fpa.org.br/sites/default/files/pesquisaintegra.pdf
12. Villar J, Carroli G, Khan-Neelofur D, Piaggio G, Gülmezoglu M. Patterns of routine antenatal care for low-risk pregnancy. Cochrane Database Syst Rev. 2001; (6):CD000934.

13. Organización Panamericana de la Salud (OPS). $L i-$ neamientos y directrices de enfermería para la mejoría de la calidad de la atención prenatal en embarazos de bajo riego en América Latina y el Caribe. Washington: OPS; 2004.

14. Calderon IMP, Cecatti JG, Vega CEP. Intervenções benéficas no pré-natal para prevenção da mortalidade materna. Rev Bras Ginecol Obstet. 2006; 28(5): 310-315.

15. Parada CMGL. Avaliação da assistência pré-natal e puerperal desenvolvidas em região do interior do Estado de São Paulo em 2005. Rev. Bras. Saude Mater. Infant. 2008; 8(1):113-124.

16. Cunha MA, Mamede MV, Dotto LMG, Mamede FV. Assistência pré-natal: competências essenciais desempenhadas por enfermeiros. Esc Anna Nery Rev Enferm 2009; 13(1):145-153.

17. Narchi NZ. Atenção pré-natal por enfermeiros na Zona Leste da cidade de São Paulo, Brasil. Rev Esc Enferm USP 2010; 44(2):266-273.

18. Narchi NZ. Exercise of essential competencies for midwifery care by nurses in São Paulo, Brazil. Mi dwifery 2011; 27:23-29.

19. Dotto LMG, Mamede MV, Mamede FV. Desempenho de competências obstétricas na admissão e evolução do trabalho de parto: atuação do profissional de saúde. Esc Anna Nery Rev Enferm 2008; 12(4):717-725.

20. Narchi NZ. Atenção ao parto por enfermeiros na Zona Leste do município de São Paulo. Rev Bras Enferm 2009; 62(4):546-551.

21. Cecatti JG. Saúde da mulher: enfoque na evidência científica para a prevenção da morbidade e mortalidade materna. Rev Bras Saúde Matern Infan 2005; 5(1):9-11.

22. Bussadori JCC. Ações da equipe de enfermagem no ciclo gravídico puerperal e as competências essenciais para a atenção qualificada ao parto [tese]. Ribeirão Preto (SP): Escola de Enfermagem de Ribeirão Preto da Universidade de São Paulo; 2009.

23. Cagnin ERG. Assistência de enfermagem à mulher no ciclo gravídico-puerperal: a realidade de Araraquara/ SP [dissertação]. Ribeirão Preto (SP): Escola de Enfermagem de Ribeirão Preto da Universidade de São Paulo; 2008.

24. Dotto LMG, Moulin NM, Mamede MV. Assistência pré-natal: dificuldades vivenciadas pelas enfermeiras. Rev Latinoam. Enfermagem 2006; 14(5):682-688. 
25. Dotto LMG, Mamede MV. Atenção qualificada ao parto: a equipe de enfermagem em Rio Branco, AC, Brasil. Rev Esc Enferm USP 2008; 42(2):331-338.

26. Esser MAMS. Atenção qualificada à mulher no parto: a realidade da assistência de enfermagem no município de Londrina, $P R$ [dissertação]. Ribeirão Preto (SP): Escola de Enfermagem de Ribeirão Preto da Universidade de São Paulo; 2010.

27. Fornazari DH. Atuação da equipe de enfermagem na assistência à mulher durante o trabalho de parto, parto e pós-parto imediato no município de Piracicaba, SP [dissertação]. Ribeirão Preto (SP): Escola de Enfermagem de Ribeirão Preto da Universidade de São Paulo; 2009.

28. Leite EPRC. A participação dos profissionais de enfermagem na assistência às parturientes no município de Alfenas, $M G$ [tese]. Ribeirão Preto (SP): Escola de Enfermagem de Ribeirão Preto da Universidade de São Paulo; 2009.

29. Nogueira LDP. Caracterização da assistência pré-natal prestada por profissionais de enfermagem na atenção qualificada ao ciclo gravídico-puerperal no município de Ribeirão Preto, SP [dissertação]. Ribeirão Preto (SP): Escola de Enfermagem de Ribeirão Preto da Universidade de São Paulo; 2010.

30. Sabino AMN. A enfermeira e a atenção pré-natal em São José do Rio Preto, SP [dissertação]. Ribeirão Preto (SP): Escola de Enfermagem de Ribeirão Preto da Universidade de São Paulo; 2008.

31. Vorpagel MGB. A participação dos profissionais de enfermagem no processo de nascimento no município de Porto Ferreira, SP [dissertação]. Ribeirão Preto (SP): Escola de Enfermagem de Ribeirão Preto da Universidade de São Paulo; 2008.

32. United Nations Population Fund (UNFPA). Maternal Mortality Update 2006. Expectation and Delivery: Investing in Midwives and Others with Midwifery Skills. New York: UNFPD; 2007. [cited 2011 Jul 5]; [about 78 p.]. Available from: http://www. unfpa.org/webdav/site/global/shared/documents/ publications $/ 2007 / \mathrm{mm}$ _update06_eng.pdf

33. United Nations Population Fund (UNFPA). Towards MDG 5: Scaling up the capacity of midwives to reduce maternal mortality and morbidity. Workshop Report. New York: UNFPA; 2006. [cited 2011 Jul 5]; [about 52 p.]. Available from: http://www. unfpa.org/webdav/site/global/shared/documents/ publications/2006/midwives_mm.pdf

34. Walsh D, Downe SM. Outcomes of free-standing, midwife-led birth centers: a structured review. Birth 2004; 31(3):222-229.
35. Hodnett Ellen D, Gates Simon, Hofmeyr G Justus, Sakala Carol, Weston Julie. Continuous support for women during childbirth. Cochrane Database of Systematic Reviews. The Cochrane Library 2011; 6(CD003766).

36. Overgaard C, Moller AM, Fenger-Gron M, Knudsen LB, Sandall J. Freestanding midwifery unit versus obstetric unit: a matched cohort study of outcomes in low-risk women. BMJ Open 2011; $1: \mathrm{e} 000262$.

37. World Health Organization (WHO). The world health report 2005: Make every woman and child count. Geneva: WHO; 2005. [cited 2011 Jul 5]; [about 243 p.]. Available from: http://www.who.int/whr/ 2005/whr2005_en.pdf

38. World Health Organization (WHO). Safe motherhood needs assessment - guidelines. Geneva: WHO; 2001. [cited 2011 Jul 5]; [about 228 p.]. Available from: http:/ /www.who.int/reproductivehealth/publications/ maternal_perinatal_health/rht_msm_96_18/en/ index.html

39. World Health Organization (WHO). Making Pregnancy Safer: the critical role of the skilled attendant. Geneva: WHO, ICM, FIGO; 2004. [cited 2011 Jul 5]; [about 18 p.]. Available from: http://www.who. int/making_pregnancy_safer/documents/924159 1692/en/index.html

40. Merighi MAB. Enfermeiras obstétricas egressas da Escola de Enfermagem da Universidade de São Paulo: caracterização e trajetória profissional [tese]. São Paulo (SP): Escola de Enfermagem da Universidade de São Paulo; 2000.

41. Merighi MAB, Yoshizato E. Seguimento das enfermeiras obstétricas egressas dos cursos de habilitação e especialização em enfermagem obstétrica da Escola de Enfermagem da Universidade de São Paulo. Rev Lat. Am. Enfermagem 2002; 10(4):493-501.

42. Riesco MLG, Teixeira BHC. Realidade e expectativas do estudante de enfermagem quanto à capacitação e qualificação na área de Enfermagem Obstétrica. Nursing 2002; 5(53):15-21.

43. Porto F, Moraes NA, Nascimento MAL. Impacto de uma portaria ministerial: aspectos da concretude social e política da enfermagem obstétrica. Rev Bras Enferm 2002; 55(4):440-443.

44. Nunes IM, Ferreira SL, Paiva MS. Condições de trabalho de enfermeiras obstetras: aspectos de uma realidade. Rev Bras Enferm 2002; 55(6):652-657.

45. Angulo-Tuesta A, Giffin K, Gama AS, D’Orsi E, Barbosa GP. Saberes e práticas de enfermeiros e obstetras: cooperação e conflito na assistência ao parto. Cad Saude Publica 2003; 19(5):1425-1436. 
46. Riesco MLG. Enfermeira obstetra: herança de parteira e herança de enfermeira. Rev Latinoam Enfermagem 1998; 6(2):13-15.

47. Riesco MLG, Tsunechiro MA, Bonadio IC. Obstetriz e enfermeira obstétrica: revendo sua formação. Acta Paul Enferm 2000; 13(Esp. pt 2):212-216.

48. Riesco MLG, Fonseca RMGS. Elementos constitutivos da formação e inserção de profissionais não médicos na assistência ao parto. Cad Saude Publica 2002; 18(3):685-698.

49. Riesco MLG, Tsunechiro MA. Formação profissional de obstetrizes e enfermeiras obstétricas: velhos problemas ou novas possibilidades? Rev. Estud. Fem 2002; 10(2):449-459.

50. Osava RH, Riesco MLG, Tsunechiro MA. Parteiras-enfermeiras e enfermeiras-parteiras: a interface de profissões afins, porém distintas. Rev Bras Enferm 2006; 59(5):699-702.

51. Merighi MAB, Gualda DMR. O cuidado à saúde materna no Brasil e o resgate do ensino de obstetrizes para assistência ao parto. Rev Latinoam Enfermagem 2009; 17(2):265-270.

52. Narchi NZ, Silva LCFP, Gualda DMR, Bastos MH Reclaiming direct-entry midwifery training in Brazil: context, challenges and perspectives. Midwifery 2010; 26(4):385-388.

53. United Nations Population Fund (UNFPA). El Estado de las Parteras en el mundo 2011. Cuidar la salud, salvar vidas. New York: UNFPA; 2011. [cited 2012 Jan 9]; [about 166 p.]. Available from: http://www. unfpa.org.ni/files/titulo/1314660602_es_SoWMy_ Full.pdf

Artigo apresentado em 15/10/2011

Aprovado em 25/01/2012

Versão final apresentada em 02/02/2012 